https://doi.org/10.15407/ujpe65.2.157

A.V. ZINOVCHUK, E.A. SEVOST'YANOV

Ivan Franko State University of Zhytomyr

(40, Velyka Berdychivs'ka Str., Zhytomyr 10008, Ukraine; e-mail: zinovchuk.a@zu.edu.ua)

\title{
INFLUENCE OF ATOMIC DISORDER ON THE AUGER RECOMBINATION RATE IN $p$-InGaN ALLOYS
}

\begin{abstract}
The influence of the atomic disorder on the Auger recombination rate in p-InGaN alloys has been studied. The disorder was simulated using a $4 \times 4 \times 4$ supercell in which In and Ga atoms taken in a required stoichiometric ratio were randomly distributed over the supercell sites. A comparison between the Auger recombination rates calculated in the framework of the supercell and virtual-crystal approximations showed that a large number of allowed interband transitions induced by the atomic disorder strongly increases the Auger recombination rate in wide-band-gap p-InGaN alloys.

Ke ywords: InGaN compounds, atomic disorder, Auger recombination, supercell.
\end{abstract}

\section{Introduction}

The $\operatorname{In}_{x} \mathrm{Ga}_{1-x} \mathrm{~N}$ compounds are widely used as radiative components in light-emitting diodes (LEDs) and visible and ultraviolet lasers [1], which is associated with the ability to vary the band gap width in those compounds within almost the whole visible spectral interval, by changing their stoichiometric composition $(x)$. The intrinsic quantum efficiency of radiating devices is determined by the rates of the radiative and non-radiative recombination processes of electrons and holes. The results of experimental researches showed that a reduction in the efficiency of $\mathrm{In}_{x} \mathrm{Ga}_{1-x} \mathrm{~N}$-based LEDs may be associated with the activation of the non-radiative Auger recombination process $[2,3]$. This is a process in which the energy emitted at the recombination of an electron and a hole is transferred to another electron or hole, which transits afterward into a high-energy state in the conduction or valence band.

A number of theoretical works devoted to the calculation of the rate of Auger processes in nitrides have been carried out till now. In particular, it was

(C) A.V. ZINOVCHUK, E.A. SEVOST'YANOV, 2020

ISSN 2071-0194. Ukr. J. Phys. 2020. Vol. 65, No. 2 demonstrated that the direct Auger recombination rate is too low to affect the efficiency of radiating devices $[4,5]$. Therefore, further efforts were focused on studying the indirect mechanisms of Auger recombination. For example, the results of calculations performed in works $[6,7]$ testify that the rate of indirect phonon-assisted Auger recombination is rather high.

The recombination owing to the presence of a disorder in the compound structure is an indirect process of another kind. When growing the $\operatorname{In}_{x} \mathrm{Ga}_{1-x} \mathrm{~N}$ compound, some Ga atoms are substituted by In ones to provide a required stoichiometric composition. The positions of substituted atoms in the compound can be regarded as almost random. As a result, unlike the case of binary compounds $\mathrm{InN}$ and $\mathrm{GaN}$, the translational periodicity of the atomic arrangement in ternary compounds becomes lost. This circumstance, in turn, gives rise to the appearance of stochastic fluctuations in the crystalline potential, which invokes an additional mechanism of free charge carrier scattering.

All above-mentioned calculations of the Auger recombination rate in nitride compounds were carried out in the virtual-crystal approximation. However, 
the latter does not make allowance for the influence of a crystal disorder on the energy band structure and the wave functions of free charge carriers. At present, we know only two papers in which the effect of a structure disorder on the Auger recombination rate in $\mathrm{In}_{x} \mathrm{Ga}_{1-x} \mathrm{~N}$ compounds was estimated numerically $[8,9]$. In work [8], a special quasirandom 32-atom $\mathrm{In}_{0.25} \mathrm{Ga}_{0.75} \mathrm{~N}$ structure was used to simulate ternary nitride compounds with an arbitrary stoichiometric composition. A more general approach was used in work [9], where every compound was modeled in the form of a large supercell with a random atomic distribution. However, the analysis in work [9] concerned only compounds of the $n$-type.

Therefore, the aim of this work is to study the effect of a disordered atomic structure in $p-\operatorname{In}_{x} \mathrm{Ga}_{1-x} \mathrm{~N}$ compounds with the crystalline lattice of the wurtzite type on the Auger recombination rate. Effects of a disordered lattice structure are simulated by considering a $4 \times 4 \times 4$ supercell containing 256 atoms. The recombination rate is calculated on the basis of the band structure and the wave functions of free charge carriers obtained in the framework of the empirical pseudopotential method. It is shown that the influence of the atomic disorder on the Auger recombination rate in nitride compounds of the $p$-type is stronger than that in the compounds of the $n$ type. This result occurs owing to the growth in the number of allowed interband Auger transitions for holes in the valence bands induced by the atomic disorder. The Auger recombination coefficient in the wide-band-gap $p$ - $\operatorname{In}_{x} \mathrm{Ga}_{1-x} \mathrm{~N}$ compounds is found to fall within the interval from $2.0 \times 10^{-32}$ to $1.0 \times$ $10^{-30} \mathrm{~cm}^{6} / \mathrm{s}$.

\section{Theoretical Model}

The supercell method is one of the widely used methods for the theoretical examination of disordered compounds [10]. A supercell is created by performing multiple and parallel translations of a unit cell along the basis vectors of the crystal. Disordered compounds are modeled by randomly filling the supercell sites with atoms of a definite kind. The larger the cell, the more adequately it describes the properties of a real compound. However, if the supercell is too large, the required calculations become very complicated and take a long time because of a large number of atoms. For available computer capabilities, the most optimal choice in our case was a $4 \times 4 \times 4$ supercell with 256 atoms. The cation sites of the supercell were randomly filled with In and Ga atoms in a way that provided the required stoichiometry of the $p-\mathrm{In}_{x} \mathrm{Ga}_{1-x} \mathrm{~N}$ compound. The lattice constants for crystals of the wurtzite type were taken as follows: $a=3.189 \AA$ and $c=5.185 \AA$ for GaN, and $a=3.544 \AA$ and $c=5.718 \AA$ for InN.

The energy band structure and the wave functions of charge carriers in the $\operatorname{In}_{x} \mathrm{Ga}_{1-x} \mathrm{~N}$ compounds were calculated using the empirical pseudopotential method which was successfully applied to binary GaN and InN compounds [11]. The set of basis functions for the pseudopotential calculation included 12483 plane waves. Such a large number of plane waves is required to provide the convergence of eigenvalues for the pseudopotential Hamiltonian. At the same time, it significantly complicates the procedure of diagonalization of the Hamiltonian when finding those values. Moreover, in order to calculate the rate of Auger recombination, the pseudopotential Hamiltonian has to be diagonalized at a rather large number of k-points in the Brillouin zone. As usual, the applied diagonalization methods are not capable of fulfilling this task. Therefore, in this work, we used the Jacobi-Davidson iteration method with a preliminary incomplete LU decomposition of the Hamiltonian [12]. This method makes it possible to calculate only the eigenvalues located within a predetermined interval. As a result, the calculation process becomes substantially accelerated in comparison with conventional methods in which absolutely all eigenvalues have to be determined. In the case of selected supercell, the time required to calculate the total band structure of the compound using a $2.6-\mathrm{GHz}$ processor was about $27 \mathrm{~h}$.

The dominant mechanism of recombination in $p$ type materials is a process with the participation of two holes and one electron (the hhe process). The rate of Auger recombination of this type can be calculated using the following formula [13]:

$$
\begin{aligned}
& R=2 \frac{2 \pi}{\hbar} \frac{V^{6}}{(2 \pi)^{9}} \iiint \int|M|^{2} f_{p}\left(E_{1}\right) f_{p}\left(E_{2}\right) f_{n}\left(E_{1^{\prime}}\right) \times \\
& \times\left(1-f_{p}\left(E_{2^{\prime}}\right)\right)\left(1-\exp \left[\left(E_{\mathrm{F} p}-E_{\mathrm{F} n}\right) / k_{b} T\right]\right) \times \\
& \times \delta\left(E_{1}+E_{2}-E_{1^{\prime}}-E_{2^{\prime}}\right) d \mathbf{k}_{1} d \mathbf{k}_{2} d \mathbf{k}_{1^{\prime}} d \mathbf{k}_{2^{\prime}},
\end{aligned}
$$

where $M$ is the matrix element of the Auger transition; $E_{\mathrm{F} n}$ and $E_{\mathrm{F} p}$ are the Fermi quasilevels of elec- 
trons and holes, respectively; $f_{n}(E)$ and $f_{p}(E)$ are the corresponding Fermi-Dirac functions; the subscripts 1,2 , and $1^{\prime}$ denote the states of holes and the electron before the recombination; the subscript $2^{\prime}$ denotes the hole state after the recombination; and $V$ is the material volume.

The matrix element of the Auger transition, $M$, is the sum of the direct, $M_{\mathrm{D}}$, and exchange, $M_{\mathrm{Ex}}$, components of the matrix element of the screened Coulomb interaction, $M=M_{\mathrm{D}}+M_{\mathrm{Ex}}$. In particular,

$$
\begin{aligned}
& M_{\mathrm{D}}=\frac{e^{2}}{V \epsilon_{0}} \sum_{G_{U}} \frac{1}{\epsilon\left(\mathbf{k}_{1}-\mathbf{k}_{1^{\prime}}+\mathbf{G}_{U}\right)} \times \\
& \times \frac{1}{\left|\mathbf{k}_{1}-\mathbf{k}_{1^{\prime}}+\mathbf{G}_{U}\right|^{2}+\lambda^{2}} \times \\
& \times \sum_{G_{1}} A_{p}^{*}\left(\mathbf{k}_{1}+\mathbf{G}_{1}\right) A_{n}\left(\mathbf{k}_{1^{\prime}}+\mathbf{G}_{1}-\mathbf{G}_{U}\right) \times \\
& \times \sum_{G_{2}} A_{p}^{*}\left(\mathbf{k}_{2}+\mathbf{G}_{2}\right) A_{p}\left(\mathbf{k}_{2^{\prime}}+\mathbf{G}_{2}-\mathbf{G}_{U}\right) \times \\
& \times \delta\left(\mathbf{k}_{1}+\mathbf{k}_{2}-\mathbf{k}_{1^{\prime}}-\mathbf{k}_{2^{\prime}}\right),
\end{aligned}
$$

where $A_{n}$ and $A_{p}$ are the coefficients in the expansion of the wave functions of electrons and holes in the plane-wave basis; summation is carried out over the vectors $\mathbf{G}_{1}, \mathbf{G}_{2}$, and $\mathbf{G}_{U}$ of the reciprocal lattice; $\epsilon\left(\mathbf{k}_{1}-\mathbf{k}_{1^{\prime}}+\mathbf{G}_{U}\right)$ is the dielectric function; and $\lambda^{2}=p e^{2} /\left(\epsilon_{0} k_{b} T\right)$ is the inverse Debye screening length which depends on the free hole concentration $p$ for materials of the $p$-type. The exchange component $M_{\mathrm{Ex}}$ can be obtained from expression (2) by swapping final states $1^{\prime}$ and $2^{\prime}$. In this work, we use the model of static dielectric function [14].

In many publications, the summation over $\mathbf{G}_{U}$ in Eq. (2) is omitted, and only the term with $\mathbf{G}_{U}=0$ is left. However, this approximation is not always justified for wide-band-gap materials. Therefore, we separately studied the dependence of the matrix element on the number of terms in the sum over $\mathbf{G}_{U}$. The calculations showed that the approximation $\mathbf{G}_{U}=0$ cannot be used for nitrides, because it leads to a significantly underestimated matrix element. To achieve the convergence in Eq. (2), the summation over at least 55 smallest $\mathbf{G}_{U}$ vectors of the reciprocal lattice has to be done.

The Fermi quasilevels are determined for a given temperature and a given free charge carrier concentration. The delta function in Eq. (1) reflects the energy conservation law at every Auger transition. In
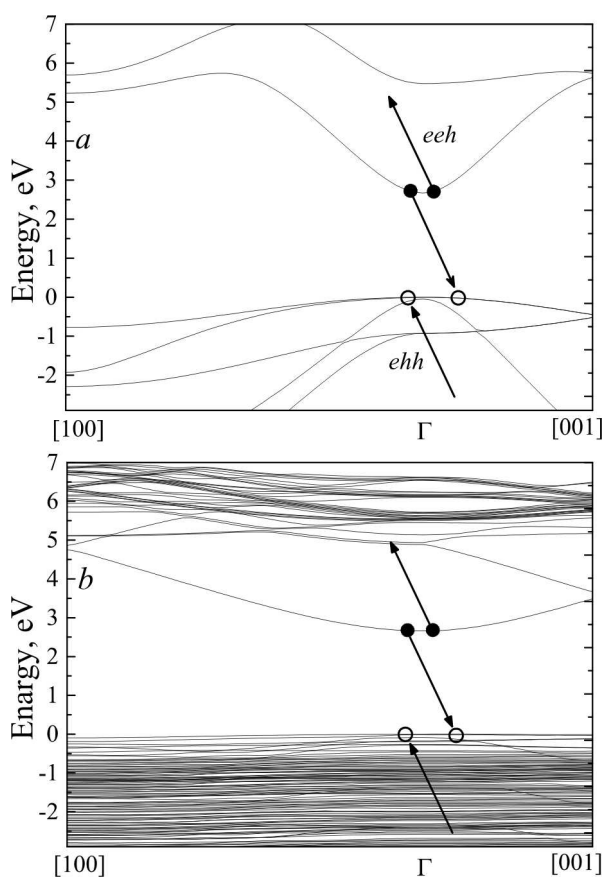

Fig. 1. Band structure of the $\mathrm{In}_{0.25} \mathrm{Ga}_{0.75} \mathrm{~N}$ compound calculated in the $(a)$ virtual-crystal and $(b)$ supercell models. Arrows indicate charge carrier transitions that take place at the Auger recombination in the $n$-type (eeh) and $p$-type (hhe) compounds

our calculations, the delta function is approximated by the Gaussian dependence with a rather small width of $0.001 \mathrm{eV}$. For the numerical integration in Eq. (1), the Brillouin zone was divided making use of a discrete system of $k$-points. The multidimensional integral was calculated using the Monte Carlo method. Test calculations showed that, in order to provide the convergence of the integration process within an accuracy of $10 \%$, a mesh of $k$-points with a density not lower than $51 \times 51 \times 51$ is required. Therefore, all the results presented below were obtained using this mesh of $k$-points.

\section{Discussion of Results}

In Fig. 1, $a$, the energy band structure of the $\mathrm{In}_{0.25} \mathrm{Ga}_{0.75} \mathrm{~N}$ compound calculated in the virtualcrystal approximation is depicted. This approximation does not account for the disordered distribution of atoms in the crystal lattice that occurs in real ternary compounds. At the same time, the calculation carried out within the supercell model (Fig. 1, b) testifies that a reduction in the translational fre- 


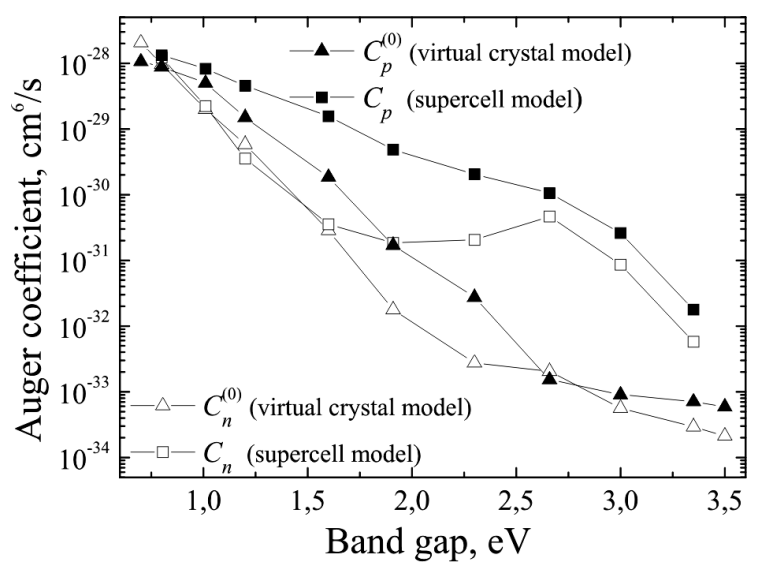

Fig. 2. Dependences of the Auger recombination coefficient on the band gap width in $p$-InGaN compounds (solid symbols) at $T=300 \mathrm{~K}$ and $n=10^{18} \mathrm{~cm}^{-3}$. Analogous dependences obtained in work [9] for $n$-InGaN compounds are also shown for comparison (hollow symbols)

quency owing to the atomic disorder leads to the formation of additional energy states both in the conduction and valence bands. As a result, the Auger transitions, which were forbidden in the virtual-crystal model, become allowed in real compounds.

For instance, the Auger transitions indicated by arrows in Fig. 1, a cannot occur both in the cases of eeh (it dominates in the $n$-type compounds) and hhe (it dominates in the $p$-type compounds) recombinations, because, in those cases, there is no finite state for the fourth charge carrier in which the conservation laws for the total energy (the delta function in Eq. (1)) and the quasimomentum (the delta function in Eq. (2)) would be obeyed simultaneously. In the supercell model, as one can see from Fig. 1, $b$, the indicated transition can take place, i.e., its probability differs from zero. Such transitions can be called indirect transitions induced by the compound disorder, similarly to indirect Auger transitions with the participation of phonons.

We also calculated the rate of Auger recombination in the virtual-crystal and supercell models. Figure 2 demonstrates the dependence of the Auger recombination coefficient on the band gap width $E_{g}$ in the $\operatorname{In}_{x} \mathrm{Ga}_{1-x} \mathrm{~N}$ compounds. The calculation was carried out for the temperature $T=300 \mathrm{~K}$ and the charge carrier concentration $p=10^{18} \mathrm{~cm}^{-3}$. The excess concentration of charge carriers was supposed to be much higher than their equilibrium concentration, $p \gg p_{0}$. We also supposed that, for the $p$-type com- pounds, $p_{0} \gg n_{0}$. Therefore, the Auger coefficient was determined from the formula $C_{p}=R / p^{3}$.

The curve with solid triangles exhibits the Auger coefficients calculated in the virtual-crystal model. In essence, this is the dependence of the coefficient of direct Auger recombination, $C_{p}^{(0)}$, on the compound band gap width. The curve with soild squares demonstrates the results of calculations in the supercell model, i.e., it displays the coefficient of indirect recombination, $C_{p}$, which accounts for the transitions associated with an atomic disorder as well.

As one can see from the comparison of those curves, the coefficients of direct and indirect recombinations differ little from each other for narrow-band-gap compounds. This is a result of the fact that only the intraband transitions are allowed (i.e. they have a non-zero probability) for such compounds. For wide-band-gap compounds, the energy and quasimomentum conservation laws can be obeyed for recombining charge carriers in the case of interband transitions as well. Due to a large amount of valence bands emerging owing to an atomic disorder, the number of possible interband transitions becomes very large in the supercell model, and the coefficient of indirect recombination $C_{p}$ is considerably higher.

The values of $C_{p}$ for InGaN compounds corresponding to the visible spectral interval $\left(E_{g}=2.6 \div\right.$ $3.3 \mathrm{eV})$ fall within the interval from $2.0 \times 10^{-32}$ to $1.0 \times 10^{-30} \mathrm{~cm}^{6} / \mathrm{s}$. The indicated values are somewhat lower than the experimental values for the Auger recombination coefficient known from the literature, $C_{p}=2.0 \times 10^{-31} \div 1.0 \times 10^{-29} \mathrm{~cm}^{6} / \mathrm{s}[15]$. We associate this difference with the indirect phonon-assisted Auger recombination, which was not taken into account in our calculations.

For the sake of comparison, Fig. 2 also demonstrates the results of calculations for the Auger recombination coefficient in $n$-InGaN, which were obtained in our work [9]. It is evident that the influence of an atomic disorder is relatively larger in the $p$-type nitrides than in the $n$-type materials. This is a direct result of the much more complicated valence band structure and, as a consequence, a larger number of allowed Auger transitions for the recombination of the hhe-type.

\section{Conclusions}

To summarize, it should be noted that the atomic disorder gives rise to a considerable growth of the 
Auger recombination rate in wide-band-gap nitride compounds of the $p$-type. The values obtained for the Auger recombination coefficients testify that this non-radiative process has to be taken into account when analyzing the causes of the efficiency reduction of nitride-based light-emitting devices. Further researches should be aimed at determining the role of indirect phonon-assisted Auger recombination in disordered nitride compounds.

1. Y. Zhao, H. Fu, G.T. Wang, S. Nakamura. Toward ultimate efficiency: progress and prospects on planar and 3D nanostructured nonpolar and semipolar InGaN lightemitting diodes. Adv. Opt. Photon. 10, 246 (2018).

2. Y.C. Shen, G.O. Mueller, S. Watanabe, N.F. Gardner, A. Munkholm, M.R. Krames. Auger recombination in InGaN measured by photoluminescence. Appl. Phys. Lett. 91, 14101 (2007).

3. J. Iveland, L. Martinelli, J. Peretti, J.S. Speck, C. Weisbuch. Direct measurement of Auger electrons emitted from a semiconductor light-emitting diode under electrical injection: Identification of the dominant mechanism for efficiency droop. Phys. Rev. Lett. 110, 177406 (2013).

4. J. Hader, J.V. Moloney, B. Pasenow, S.W. Koch, M. Sabathil, N. Linder, S. Lutgen. On the importance of radiative and Auger losses in GaN-based quantum wells. Appl. Phys. Lett. 92, 261103 (2008).

5. F. Bertazzi, M. Goano, E. Bellotti. Auger recombination in GaInN/GaN quantum well laser structures. Appl. Phys. Lett. 97, 231118 (2010).

6. F. Bertazzi, M. Goano, E. Bellotti. Numerical analysis of indirect Auger transitions in InGaN. Appl. Phys. Lett. 101, 011111 (2012).

7. E. Kioupakis, P. Rinke, K.T. Denaley, C.G. Van de Walle. Indirect Auger recombination as a cause of efficiency drop in nitride light-emitting diodes. Appl. Phys. Lett. 98, 161107 (2011).

8. E. Kioupakis, D. Steiauf, P. Rinke, K.T. Delaney, C.G. Van de Walle. First-principles calculations of indirect Auger recombination in nitride semiconductors. Phys. Rev. B 92, 035207 (2015).

9. A.V. Zinovchuk, A.M. Gryschuk. Alloy-assisted Auger recombination in InGaN. Opt. Quant. Electron. 50, 455 (2018).
10. V. Popescu, A. Zunger. Extracting $E$ versus $\mathbf{k}$ effective band structure from supercell calculations on alloys and impurities. Phys. Rev. B. 85, 085201 (2012).

11. M. Goano, E. Bellotti, E. Ghillino, G. Ghione, K. Brennan. Band structure nonlocal pseudopotential calculation of the III-nitride wurtzite phase materials system. Part I. Binary compounds GaN, AlN, and InN. J. Appl. Phys. 88, 6467 (2000).

12. A.R. Tackett, M. Di Ventra. Targeting specific eigenvectors and eigenvalues of a given Hamiltonian using arbitrary selection criteria. Phys. Rev. B 66, 245104 (2002).

13. D.B. Laks, G.F. Neumark, S.T. Pantelides. Accurate interband-Auger-recombination rates in silicon. Phys. Rev. B 42, 5176 (1990).

14. G. Cappellini, R. Del Sole, L. Reining, F. Bechstedt. Model dielectric function for semiconductors. Phys. Rev. B 47, 9892 (1993)

15. J. Piprek, F. Romer, B. Witzigmann. On the uncertainty of the Auger recombination coefficient extracted from InGaN/GaN light-emitting diode efficiency droop measurements. Appl. Phys. Lett. 106, 101101 (2015).

Received 01.07.19. Translated from Ukrainian by O.I. Voitenko

\section{А.В. Зіновчук, Є.О. Севостьлнов}

ВПЛИВ НЕВПОРЯДКОВАНОЇ

АТОМНОЇ СТРУКТУРИ НА ШВИДКІСТЬ

ОЖЕ-РЕКОМБІНАЦЇ̈ В InGaN СПОЛУКАХ $p$-ТИПУ

$\mathrm{P}$ е 3 ю м е

Було досліджено вплив атомної невпорядкованості на швидкість оже-рекомбінації в $p$-InGaN сполуках. Моделювання невпорядкованості виконувалось за допомогою $4 \times$ $\times 4 \times 4$ надкомірки, в якій In та Gа атоми випадковим чином розміщувалися по вузлах, забезпечуючи необхідний стехіометричний склад сполуки. Порівняння швидкості оже-рекомбінації, розрахованої в межах апроксимації надкомірки та апроксимації віртуального кристала, показує, що велика кількість дозволених міжзонних оже-переходів, спричинених атомною невпорядкованістю, значно підвищує швидкість рекомбінації в широкозонних InGaN сполуках $p$-типу. 